\title{
SISTEMA DE CLASSIFICAÇÃO DE PACIENTES - PARTE I: DIMENSIONAMENTO DE PESSOAL DE ENFERMAGEM
}

Jose Rodrigues Filho*

RODRIGUES FILHO, J. Sistema de classificaçăo de pacientes - Parte I: dimensionamento de : pessoal de enfermagem. Rev.Esc.Enf.USP, v.26, n.3, p. $\quad-\quad$, dez., 1992.

Um sistema de classificaçăo de pacientes pode ser considerado como um instrumento essencial da pratica administrativa na drea de enfermagem. Estes sistemas proporcionam aos gerentes de enfermagem informaçdes para o processo de tomada de decisdo em áreas referentes d alocaçdo de recursos humanos, monitoramento da produtividade e custos de enfermagem. $O$ proposito deste trabalho é o de demonstrar a importancia de um sistema de classificaçdo de pacientes, depois de uma revisdo da literatura, com a esperança de que este tópico venha a fazer parte de uma agenda de pesquisa no Brasil.

UNITERMOS: Cuidados de enfermagem. Sistema de classificação de pacientes. Recursos humanos em enfermagem.

\section{Introduçáo}

Em recente trabalho realizado no Hospital Universitário Lauro Wanderley, da Universidade Federal da Parafba, destinado, em parte, a dimensionar o quantitativo de pessoal de enfermagem, foi constatado que qualquer tentativa de dimensionamento de pessoal, com base na hora média de assistencia de enfermagem e o número de leitos ocupados, conforme estabelece a literatura nacional, e um exercício incompleto, obsoleto e enviesado. A conclusão do trabalho enfatizou a necessidade de se criar parâmetros reais, apos atritos com algumas chefes de unidades de enfermagem que, defendendo o status quo, também năo ofereciam argumentos válidos para se determinar o staff de enfermagem das várias unidades hospitalares(21).

Este fato contribuiu para que o autor do presente trabalho fizesse uma revisåo da literatura sobre tåo importante tópico, na expectativa de que seja determinada uma agenda de pesquisa na área de enfermagem, no Brasil, para tratar este assunto de maneira científica, a exemplo do que vem acontecendo nos países desenvolvidos.

Embora um dos grandes problemas dos administradores hospitalares e da gerência de enfermagem seja o de determinar o quantitativo de pessoal neces-

- Pesquisador e Takemi Fellow na Universidade de Harvard. Professor do Departamento de Administração da Universidade Federal da Paraíba/UFPb. 
sário para atender aos cuidados de saúde de pacientes, no Brasil são quase inexistentes pesquisas nesta área. Segundo a literatura nacional, o enfoque utilizado e destinado a calcular o pessoal de enfermagem na área hospitalar se assemelha àquele utilizado nos Estados Unidos na década de 1950.

Isto é demonstrado em estudo destinado a calcular o pessoal de enfermagem do Hospital Universitário da Universidade de São Paulo. Os autores desse estudo concluiram, portanto, que "os resultados apresentados evidenciam uma variaçăo entre os índices citados e os dados reais do HU", propondo que uma classificação de pacientes, por tipo de cuidado, tornaria "o cálculo de pessoal mais próximo da necessidade real" (3).

Portanto, a criação de um sistema de classificação de pacientes, como ferramenta da prática administrativa de enfermagem, proporciona a formação de um banco de dados indispensável ao processo de tomada de decisão em áreas relacionadas com o dimensionamento e alocação de recursos humanos, qualidade do cuidado médico, monitoramento da produtividade e custos dos serviços de enfermagem.

O presente trabalho, portanto, é o primeiro de uma série de três, destinado a enfocar a importância da criação de um sistema de classificação de pacientes, como base na literatura revisada, como instrumento gerencial de enfermagem tanto para o dimensionamento do quantitativo de pessoal como para elaboraçáo de sistemas de custos condizentes com a prática de enfermagem. Posteriormente, a importáncia de um sistema de classificação de pacientes será analisada sob a perspectiva de custos dos serviços de enfermagem e de aplicativos computacionais nesta área.

\section{Histórico}

Embora a história sobre classificação de pacientes na área de enfermagem tenha surgido desde o período de Florence Nightingale, o desenvolvimento de sistemas de classificação de pacientes teve início a partir do efeito de um estudo realizado em 25 hospitais de Nova York, Estados Unidos, na década de 1930. Este estudo é considerado como uma das primeiras tentativas de se identificar as necessidades de paciemtes de maneira quantificável, operacional e compreensiva, já que recomendava uma média de três horas e meia $(3,5)$ de cuidados de enfermagem por paciente-dia(13). Este indicador de tempo para o cuidado de enfermagem foi adotado por muitos hospitais que, infelizmente, não levaram em consideração as recomendações que sugeriam a detérminação de indicadores com base nos níveis de cuidados nas diversas unidades hospitalares. Em resumo, o indicador encontrado não era exato, mas oferecia subsídios para futuros estudos.

Assim sendo, a noção de classificação de pacientes foi considerada num trabalho realizado pela Escola de Enfermagem de Pittsburgh, em 1950, destinado a determinar as exigências de cuidados de enfermagem para pacientes com diagnósticos de clínica médica e cirúrgica(12). Em 1951, o serviço militar americano iniciou experimentos na área de classificação de pacientes, aceitando um instrumento de classificação de cuidados de enfermagem de 4 (quatro) categorias(6).

Contudo, desenvolvimento no campo de classificação de pacientes, nos 
Estados Unidos, ganhou ritmo acelerado a partir da década de 1960, após estudos realizados por um grupo de pesquisadores da Escola de Engenharia e da Divisăo de Pesquisa Operacional do Hospital da Universidade Johns Hopkins(7). Conseqüentemente, vários trabalhos destinados a dimensionar o staff de enfermagem na área hospitalar, de forma racional, utlizando técnicas do campo da engenharia industrial e da ciência da administração foram desenvolvidos tanto nos Estados Unidos como na Grã-Bretanha(1,2,11,16,22,23,24).

Na década de 1970 surgiram outros sistemas de classificação de pacientes na área de enfermagem bastante reconhecidos(3,9). Segundo Giovanneti, cerca de 1.000 hospitais americanos estavam utilizando algum tipo de sistema de classificação de pacientes no final da década de 1970(14).

Estes instrumentos de classificação de pacientes adotavam uma das se-guintes estruturas básicas: 1) um instrumento denominado de "protótipo", com três ou quatro categorias de pacientes, com a descrição das exigências do cuidado de enfermagem em cada categoria; 2 ) outro instrumento denominado de wavaliaçăo de fatores", envolvendo uma lista de "indicadores críticos" das diferentes exigencias do cuidado de enfermagem, considerados separadamente. A soma dos indicadores indicava a categoria do paciente. $O$ segundo instrumento é mais utilizado já que, no primeiro, surgem dificuldades quando o paciente tem suas necessidades de cuidados nas diferentes categorias. Quanto ao segundo instrumento, surge também a seguinte questão: São os indicadores representativos das necessidades de cuidado de enfermagem?

Os dois tipos de classificação são também considerados como "subjetivo" (protótipo) e "objetivo" (avaliação de fatores), embora esta terminologia seja inadequada, uma vez que alguma medida de subjetividade é inevitável em qualquer avaliação das exigências do cuidado de enferrmagem(14).

Na década de 1980, as limitações de alguns sistemas de classificação de pacientes, desenvolvidos principalmente na década de 1960, começaram a ser re-conhecidas nos Estados Unidos(8,15). Tais sistemas tiveram como objetivo principal determinar o staff de enfermagem, ou seja, o resultado de tais instrumentos era o de projetar a demanda de tempo de cuidado de enfermagem.

Hoje, porém, com o avanço das teorias científicas, sistemas de informações e as restriçōes económicas, os objetivos destes sistemas são mais amplos. Desta forma, os métodos tradicionais de classificação de pacientes estão sendo reconhecidos como inadequados para atender a sofisticação e complexidade dos objetivos atuais.

Portanto, objetivando atender as necessidades atuais, um novo grupo de pesquisadores da Universidade de Johns Hopkins, orientados pelo trabalho da professora Susan Horn, daquela Universidade, começaram recentemente a desenvolver um novo sistema de classificação de pacientes para a enfermagem, com base num índice de severidade computadorizado, criado há pouco tempo $(17,18)$.

Este novo sistema de classificaçāo pode se tornar universal, já que oferece a capacidade de prever as exigências ou demanda de cuidado de enfermagem, além de ser considerado clinicamente significativo, objetivo, confiável, fácil de ser atualizado, oferecendo medidas válidas das necessidades do paciente, além de permitir comparação entre hospitais. 
Um sistema de classificação de pacientes para a enfermagem pode ser definido como o que determina a demanda ou exigências do paciente em termos de cuidados de enfermagem, com base nas suas necessidades, nas intervenções apropriadas da enfermagem e nas prioridades do cuidado. Em resumo, um sistema de classificação se refere a um conjunto de pacientes que seja homogêneo em termos do consumo do cuidado de enfermagem.

Assim sendo, o resultado de um sistema de classificação de pacientes é o nível de cuidado, que nos capacita a reconhecer a variância de severidade (nível de cuidado) entre diferentes pacientes ou a variância do cuidado de um único paciente entre diferentes dias de hospitalização. Por outro lado, um sistema de dimensionamento de pessoal de enfermagem tem como resultado a identificação de padrões de pessoal, exigidos para a prestação do cuidado de saúde.

A falta de claridade ou utilização de um enfoque sistêmico contribuiu para criar confusão na utilização dos sistemas de classificação de pacientes, que hoje sofrem algumas limitaçóes como:

1 - os sistemas atuais não retêm (capturam) características especificas do paciente em termos da doença e sua extensão de distúrbios fisiológicos;

2 - muitos sistemas são baseados em tarefas desenvolvidas pela enfermagem para o cuidado do paciente, em vez de serem baseados nas necessidades objetivas do paciente;

3 - estes sistemas não são testados nem comparados, embora as atividades e até privilégios da enfermagem variem de região para região ou de instituiçăo para instituiçăo;

4 - os critérios de validade e confiabilidade destes sistemas são criticados, já que são muitas vezes subjetivos e não sensíveis às características do paciente;

5 - a inexistência de um padrāo de aceitação mínimo e universal de cuidado de enfermagem, componente principal de um sistema de classificaçăo de pacientes na área de enfermagem.

Algumas exigências são necessárias para o desenvolvimento de um sistema de classificaçăo de pacientes na área de enfermagem. Para De Groot alguns componentes ou elementos devem ser incluídos num sistema de classificação de pacientes, a saber(8):

1 - um instrumento para prever as exigéncias de cuidados de enfermagem, permitindo variações das características do paciente; 
2 - um método destinado a validar o volume de cuidados dado a cada categoria de pacientes, em cada unidade ou turno;

3 - um método para avaliar os padrões de cuidados prestados por cada unidade ou turno;

4 - um método destinado a relacionar as exigéncias de cuidados de enfermagem com a alocação de recursos humanos, em cada unidade ou turno;

5 - um método destinado a monitorar a confiabilidade do sistema de classificação de pacientes ao longo do tempo.

Os sistemas de classificação de pacientes sempre foram utilizados com algum tipo de quantificação - uma estimativa dos recursos de enfermagem associada com cada categoria de cuidado. Em geral, dois métodos de quantificaçăo são utilizados: um baseado no tempo médio de cuidado para cada categoria de paciente; outro baseado no tempo padráo de cuidado para procedimentos específicos de enfermagem. No primeiro caso, o consumo médio do cuidado direto de enfermagem prestado em cada categoria é determinado através de estudos de observações. Neste caso, o número de pacientes em cada categoria multiplicado pelo correpondente tempo médio de cuidado direto nos dá uma estimativa do total do tempo médio de cuidado direto exigido. Este número adicionado a uma estimativa média de tempo inerente ao cuidado indireto (também encontrado através de estudos de observação) nos dá a média de tempo exigido para o cuidado de enfermagem para um grupo específico de pacientes.

No segundo método, um tempo padrão é calculado para cada atividade de enfermagem. O tempo de cuidado direto $\hat{e}$, portanto, determinado através da multiplicação do número de ocorrências de cada atividade pelo tempo padrão apropriado. O total de tempo para o cuidado de enfermagem é, entáo, determinado pelo adicionamento de um coeficiente representando o cuidado indireto.

O que importa nestes instrumentos de classificação são as técnicas de coleta de dados utilizadas e a forma como os estudos de observação são considerados. Embora um determinado instrumento de classificação possa ser aplicável para uma variedade de instituições, os coeficientes de quantificação não são transferíveis. $O$ tempo de cuidado médio ou tempo padrão não são necessariamente os mesmos de instituição para instituição ou mesmo de uma unidade de enfermagem para outra.

Portanto, a aplicação indevida de um tempo utilizado para o dimensionamento de pessoal do Hospital Universitário Lauro Wanderley, da Universidade Federal da Paraíba, determinado há muito tempo, em algum lugar, foi considerado suspeito. Quando tal indicador se acomodava com o atual status quo, os chefes de enfermagem pareciam satisfeitos. Porém, quando contrariava os interesses do status quo, a enfermagem considera tal indicador inaceitável para a "realidade do HU".

A condução de estudos de observação exige conhecimento e experiência quanto ao uso de métodos e técnicas necessários. Os dados obtidos através destas técnicas de quantificação se relacionam diretamente com a prática, procedimen- 
tos e o comportamento existentes durante o período de observação. Práticas ineficientes devem ser identificadas e corrigidas e os coeficientes de tempo médio do cuidado ou tempo padrão devem ser ajustados de forma apropriada, para evitar a perpetuação de erros.

As propriedades de confiabilidade e validade são essenciais para o desenvolvimento de um sistema de classificação de pacientes. Confiabilidade se refere à consistência, estabilidade ou repetitividade de um instrumento de medida. Já que o ato de classificar pacientes de acordo com as exigências do cuidado de enfermagem pode ser realizado por diferentes enfermeiras, em diferentes unidades de enfermagem, $e$ importante que seja consistente a classificação realizada por estas enfermeiras. Assim sendo, a confiabilidade deve ser constantemente monitorizada tanto dentro como entre unidades hospitalares.

O conceito de validade se refere a questáo: Estamos medindo o que nos propomos a medir? Assim, um instrumento destinado a validar o volume de cuidado dado a cada categoria ou tipo de paciente, em cada turno e em cada unidade, é considerado como o segundo elemento essencial de um sistema de classificação de pacientes.

O eue tradicional de se determinar o nível de cuidado do paciente, componente principal de um sistema de classificação de pacientes, define a enfermagem como a implementação de uma série de tarefas ou atividades (indicadores críticos). Este enfoque de tarefas medidas (indicadores críticos) evita que o sistema retenha os serviços de enfermagem de forma apropriada. Como exemplo, podemos citar o caso em que o tempo da tarefa de um banho do paciente seja superior ao tempo dado a tarefa de planejar a alta do paciente. Evidentemente, um banho pode gastar mais tempo. Contudo, como um serviço, não tem o mesmo risco, complexidade ou exigencia de um nível de habilidades que o planejamento de uma alta hospitalar exige.

Já que a demanda do cuidado de enfermagem é de caráter multidimensional, um sistema de classificação de pacientes destinado a capturar os serviços de enfermagem deve basear-se em interações complexas de fatores clínicos objetivos e subjetivos.

\section{Sistema de dimensionamento de pessoal de enfermagem}

Como se viu, um sistema de classificação de pacientes e um sistema de dimensionamento de pessoal produzem resultados distintos: nível de cuidado e padrões de pessoal. O reconhecimento destes distintos resultados oferece a enfermeira dados independentes, refletindo as exigências do paciente e as exigências de alocação de recursos humanos.

Neste sentido, esta diferenciação permite uma identificação objetiva, análise e avaliação das decisões que diretamente têm impacto sobre o cuidado do paciente sobre a alocação ảe recursos de enfermagem. É no sistema de dimensionamento de pessoal que se torna possível capturar todas as atividades de enfermagem, as quais incluem o cuidado direto, cuidado indireto e outras atividades relacionadas com a unidade de trabalho. 
Existem uma multiplicidade de fatores que afetam a determinação de cuidados tais como o tipo da organização da enfermagem, o desenho físico da área de trabalho, modalidades de tratamento, prática médica, disponibilidades das habilidades de enfermagem e até atitudes do pessoal. Assim sendo, as instituiçóes interessadas em utilizar um sistema de classificação de pacientes necessitam determinar seu proprio índice de quantificação da carga de trabalho da enfermagem.

A importância da diferenciação entre o nível de cuidado do paciente e o pessoal de enfermagem será explorada a seguir, com algumas questões:

1 - É a prática pré-determinada do pessoal de enfermagem que direciona a determinação do nivel de cuidado ou são os niveis de cuidado que determinam a alocaçăo de pessoal?

A experiência tem mostrado que, frequientemente, dados referentes aos cuidados de enfermagem não são utilizados para alocar recursos humanos, embora, com se viu, os níveis de cuidados sejam o componente principal para se criar um sistema de classificação de pacientes. Em muitos casos, o sistema de classificação de pacientes torna-se inadequado quando a alocação pré-determinada de pessoal não está apoiada em níveis de cuidados quantificativos. Muitos sistemas de classificação de pacientes, por exemplo, determinam que os dois niveis mais elevados do cuidado de enfermagem sejam utilizados para unidades de tratamento intensivo. A explicação é que estas unidades devem ser, em geral, dotadas de uma enfermeira para cada paciente ou para dois pacientes. Neste caso, o sistema é elaborado no sentido de que é a prática do pessoal de enfermagem que determina o nível de cuidado. As evidências sugerem, contudo, que pacientes internados nestas unidades consomem uma variedade de níveis de cuidados. Sabe-se que existem muitas atividades de enfermagem que podem ser consideradas como "rituais de enfermagem" pelo fato de estarem sendo realizadas há muitos anos.

2 - Os padrões estabelecidos para o pessoal necessário ao atendimento dos niveis de cuidados não devem levar em conta todas as variáveis que deveriam ser consideradas num sistema de dimensionamento de pessoal.

É necessário reconhecer que um sistema de dimensionamento de pessoal, além de estabelecer os padrōes de pessoal para cada nível de cuidado, deve-se estabelecer padrōes para situações que não são retidas pelos dados dos níveis de cuidados. Os padrōes de pessoal para um nivel de cuidado de um determinado paciente inclui apenas os recursos apropriados para o cuidado específico deste paciente. Como exemplo, supõe-se que tenha sido atribuído a José da Silva um nível de cuidado 3 (três) e que o padrão de pessoal para o nível de cuidado 3 seja de 6,5 horas. A enfermeira de José da Silva recebeu mais três pacientes admitidos, envolvendo um consumo de tempo maior. É necessário reconhecer que estas atividades adicionais a serem desenvolvidas pela enfermeira de José da Silva não são o resultado de uma mudança do nível de cuidado de José da Silva ou uma indicação de que o padrão de pessoal para o nível de cuidado 3 seja inapropriado. Portanto, esta situação é uma ilustração de apenas uma entre muitas variáveis a serem consideradas no desenvolvimento da política de alocação de recursos humanos. 
Alguns dos métodos utilizados para se estimar o número e espécies de pessoal de enfermagem exigidos num hospital são: 1) descritivo, 2) administração da produçáo, e 3 ) pesquisa operacional.

A metodologia descritiva utiliza dados sobre um grande número de variáveis, cuja interação entre elas não é clara. Neste caso, utiliza-se o número de horas de cuidados, proporção de pessoal e dados do censo hospitalar como pacientesdia, admissðes e altas.

A metodologia da administração da produção utiliza métodos da engenharia industrial, que é muito popular na área hospitalar. É dirigida para o estudo do trabalho das unidades de enfermagem ou de departamentos especificos. Concentra-se na identificação de tarefas, fluxo, organizaçăo e simplificaçăo do trabalho.

A metodologia de pesquisa operacional se baseia em modelos matemáticos, destinados a modelar problemas da vida real, utilizando dados do mundo real e explorando procedimentos de se alcançar soluções ótimas.

\section{Conclusão}

Um sistema de classificação de pacientes deve ser considerado como um instrumento indispensável à gerência de enfermagem. Se desenvolvido apropriadamente torna-se uma ferramenta importante tanto para a determinação e alocação de pessoal de enfermagem, como para determinação de custos, planejamento orçamentário e determinação da qualidade de cuidado médico.

Já que no Brasil não é conhecido o uso deste instrumento gerencial na rede hospitalar, é necessário que pesquisas sejam realizadas para iniciar o desenvolvimento de sistemas de classificação de pacientes, no sentido de dotar os gerentes de sistemas gerenciais modernos. Como se poderá ver, posteriormente, é através destes instrumentos que a enfermagem terá condições de determinar os custos dos serviços produzidos. A determinação do custo de enfermagem, além de ser importante sob o aspecto gerencial, é importante para fortalecer o reconhecimento e o mérito da profissão de enfermagem, que compõe o setor responsável por uma grande parcela dos gastos de saúde de qualquer país. A enfermeira norte-americana, Lynda Carpenito, defendendo recentemente um sistema de classificação com base no diagnóstico de enfermagem disse que é através do esclarecimento do significado da enfermagem para os enfermeiros, para os empregadores e o público em geral que a profissão se fortalece e enriquece(4).

Embora o governo federal tenha determinado um sistema de classificação de pacientes para efeito de pagamento hospitalar, é lamentável que no Brasil, tanto na área médica como na área de enfermagem, não tenha surgido algum tipo de sistema de classificação de pacientes $(19,20)$. Já que o mundo desenvolvido caminha para a adoção de sistemas de classificação de pacientes para serem utilizados tanto como métodos de pagamento como instrumento gerencial, destinado principalmente às atividades de planejamento e controle, espera-se que no Brasil alguns avanços sejam alcançados num futuro próximo(10).

Nesta primeira parte do trabalho é lançado um grande desafio para os administradores de enfermagem no Brasil que, sem dúvida, estão carentes de instrumentos gerenciais baseados em técnicas científicas modernas. Contudo, pelo 
pioneirismo desta profissão espera-se que num futuro próximo este tópico venha a fazer parte de uma agenda de pesquisa não só nos cursos de pós-graduaçăo como nas instituições onde a prática de enfermagem é exercida.

RODRIGUES FILHO, J. Patient classification systems: Part I - nursing personnal determination. Rev.Esc.Enf.USP., v.26, n.3, p - , dec., 1992.

A classification system can be an essential management tool of nursing administrative practice. This system should exist to provide nursing with useful and meaningful information for decisions about staffing allocations, monitoring productivity, and costing of nursing services. The purpose of this study is to show the importance of a patient classification system, after a review of current literature, in the hope that a reasearch agenda on the topic can be established in Brasil.

UNITERMS: Nursing care. Classification systems. Care requeriments. Staffing.

\section{REFERENCIAS BIBLIOGRÁFICAS}

1. BARR, A.; MOORES, B.; RHYS-HEARN, C. A review of the various methods of measuring the dependency of patients on nursing staff. Int.J.Nurs.Stud, v.10, n.3, p.195-208, 1973.

2. BARTSCHT, K.G. An analitycal approach to nursing scheduling. Hosp.Top., v.41, n.9, p.44-5. 1963.

3. CAMPEDELLI, M.C.; TAKITO, C.; SANCINETTI, T.R.; BENKO, M.A. Cálculo de pessoal de enfermagem: competencia da enfermeira. Rev.Esc.Enf.USP, v.21, n.1, p.3-15, 1987.

4. CARPENITO, L.J. Developments in nursing classification. In: AMERICAN NURSES ASSOCIATION. Classification syszems for describing nursing practice: working papers. Kansas City, 1989. (Publ. NP. 74).

5. CLARK, E.; DIGGS, W. Quantifying patient care needs. Hospitals, v.48, n18, p.96-100, 1971.

6. CLAUSSEN, M.E. Categorization of patients according to nursing care needs. Milit.Med, v.116, p.209-14, 1955.

7. CONNOR, R.J. Effective use of nursing resources: a research report. Hospitals, v.35, n.9, p.30-9, 1961.

8. DE GROOT, H.A. Patient classification system evaluation. Part I: essencial system elements. J.Nurs. Adm, v.19, n.6, p.30-5, 1989.

9. DES ORMEAUX, S.P. Implementation of the C.A.S.H.: patient classification system for sttafing determination. Superv. Nurse, v.8, n.4, p.28-30, 1977.

10. FETTER, R.B.; SHIN, Y.; FREEMAN, J.L; AVERILL, R.F.; THOMPSON, J.D. Case mix definitions by diagnosis-related groups. Med Care, v.18, p1-53. Supplement 2.

11. FLAGE, C.D. The problem of organization for hospital inpatient care. In: CHURCHMAN, C.W.; VERHULST, M. Management sciences: models and techniques. New York, Pergamon Press, 1960. v.2, cap.25, p.275-87, 1960.

12. GEORGE, F.L.; KUEHN, R.P. Patterns of patient care. New York, Macmillan, 1985.

13. GIOVANNETI, P. Patient classification systems in nursing: a description and analysis. Hyatssville, US Department of Health, Education, and Welfare. 1978. (Publication HRA 78-22, HRPO-500-501).

14. GIOVANNETI, P. Understanding patient classification systems. J.Nurs.Adm, v.9, n.2, p.4-9, 1979.

15. HAAS, S.A. Patient classification systems: a self-fulfilling prophecy. Nurs.Manage, v.19, n.5, p.56-62, 1988.

16. HAUSEN, K.E. How to measure nursing care time. Mod.Hosp., v.100, n.4, p.93-6, 1963. 
17. HORN, S.D. Severity indices: potencial uses in quality measurement. Top. Health Rec. Manage, v.10, n.2, p.45-55, 1989.

18. HORN, S.D.; HORN, R.A. Reliability and valitidy of the severity of illness index. Med Care, v.24, n.2, p.159-78, 1986.

19. RODRIGUES FILHO, J. Hospital utilizations and reimbursement method in Brazil. Int.J.Health Plann.Manage, v.4, n.1, p.3-15, 1989.

20. RODRIGUES FILHO, J. Método de pagamento hospitalar no Brasil. Rev.Adm.Públ., v.24, n.4, p.78-86, 1990.

21. RODRIGUES FILHO, J.; LOPES, M.A.A.; SANTOS, S.R. dos. Avalią̧ão da distribuição de recursos humanos no Hospital Universitário Lauro Wanderley da Universidade Federal da Parafba: relatório. . Joảo Pessoa, 1990, v.1.

22. STEINEY, B.H.; LIDQUIST, N.E. Surprise find of staffing study: nurses have too much spare time. Mod Hosp., v.114, p.108-10, 1970.

23. STIMSON, D.H.; STIMSON, R.H. Operations research in hospitals: diagnosis and prognosis, Hospital Research and Educational Trust, Chicago, 1972.

24. WOLFE, H.; YOUND, J.P. Staffing the nursinf unit, Part I: controlled variable staffing. Nurs.Res, v.14, n.3, p.236-43, 1965. 